\title{
AVANCES EN LA SELECCIÓN DE FUENTES DE RESISTENCIA A LAS PRINCIPALES ENFERMEDADES DEL FRIJOL COMÚN (Phaseolus vulgaris L.) EN COSTA RICA ${ }^{1}$
}

\author{
Carlos M. Araya ${ }^{2}$, Rodolfo Araya ${ }^{3}$
}

\begin{abstract}
RESUMEN
Avances en la selección de fuentes de resistencia a las principales enfermedades del frijol común (Phaseolus vulgaris L.) en Costa Rica. En esta investigación se evaluaron diversos genotipos de frijol en la búsqueda de fuentes de resistencia a la antracnosis (Colletotrichum lindemuthianum) y a la mancha angular (Phaeoisariopsis griseola). Se evaluó germoplasma del programa nacional de hibridaciones y de tres viveros internacionales distribuidos por el CIAT: VIFURE, VIPADOGEN y CORE COLLECTION. Los ensayos se establecieron en Puriscal (1017 msnm), Alajuela (814 msnm) y Fraijanes (1650 msnm), durante los ciclos agrícolas 97-98 y 98-99. Los materiales se sembraron en parcelas de un surco de dos a seis metros de largo con una o dos repeticiones y manejo con mínimos insumos. Se evaluó la reacción a la infección natural por antracnosis y mancha angular a las seis y ocho semanas después de la siembra, con base en la escala de severidad de uno a nueve. Se seleccionaron materiales con reacción de uno hasta grado tres para antracnosis y de uno hasta grado cuatro para mancha angular. Del grupo de materiales provenientes del programa de hibridaciones se seleccionaron nueve líneas por su resistencia a ambos patógenos. Posteriormente, sólo cinco mostraron resistencia específica a antracnosis y dos a mancha angular. De los viveros distribuidos por el CIAT, el VIPADOGEN aportó dos líneas que mostraron resistencia combinada a las dos enfermedades, mientras que 25 fueron resistentes exclusivamente a antracnosis, además de mostrar tolerancia a baja fertilidad o a sequía. Del vivero CORE COLLECTION, sólo 26 líneas mostraron resistencia combinada; 82 líneas fueron resistentes a antracnosis y 12 a mancha angular. Las mejores fuentes de resistencia a antracnosis y mancha angular estarán disponibles en un vivero internacional.
\end{abstract}

\begin{abstract}
Advances in the selection of resistance sources to important diseases common bean (Phaseolus vulgaris L.) in Costa Rica. In this research a broad set of bean genotypes were evaluated in the search for sources of resistance to both, anthracnose (Colletotrichum lindemuthianum) and angular leaf spot (Pahaeoisriopsis griseola). The germplasm evaluated came from the National Bean Breeding Program and international nurseries from CIAT: VIFURE, VIPADOGEN and CORE COLLECTION. Experimental plots were establised in Puriscal (1017 masl), Alajuela (814 masl) and Fraijanes (1650 masl), during the 97-98 and 98-99 growing seasons. Experimental plots were single or two rows from two to six $\mathrm{m}$ in length depending on seed availability. Disease reaction was scored at six and eight weeks after seeding using a 1 to 9 severity scale. Only genotypes showing 1-3 disease reaction for anthracnose and 1-4 for angular leaf spot were selected. In the germplas from the National Bean Breeding Program nine lines resistant to both pathogens were identified, of those, five lines were specifically resistant to anthracnose and two angular leaf spot. In the VIPADOGEN from CIAT, 25 lines were resistant to anthracnose and only two were resistant to both diseases. These materials also displayed adaptation to either drought or low soil fertility. The CORE COLLECTION nursery provided 82 lines resistant to anthracnose, 12 lines resistant to angular leaf spot and 26 lines were resistant to both pathogens. The most outstanding sources of resistance to anthracnose and angular leaf spot will be made available in a regional nursery.
\end{abstract}

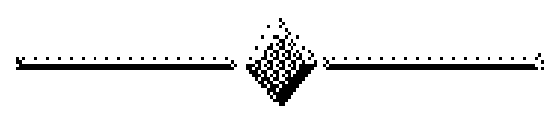

\section{INTRODUCCIÓN}

En América se han identificado dos centros de domesticación del frijol común (Phaseolus vulgaris L.): la Zona Andina y Mesoamérica. Esta domesticación ocurrió como dos procesos independientes pero simultá- neos (Gepts y Debouck, 1991). Estudios posteriores (Singh et al., 1991) han demostrado que en cada uno de esos centros han surgido tres razas de frijoles cultivados, las que presentan una gran variabilidad morfológica, bioquímica y agronómica. Esa amplia variabilidad genética le ha permitido a este cultivo adaptarse a dife-

\footnotetext{
1 Trabajo financiado parcialmente por Programa Cooperativo Regional de Frijol para Centroamérica, México y El Caribe [PROFRIJOL], Universidad Nacional y Vicerrectoría de Investigación de la Universidad de Costa Rica, proyecto 736-91-315 y la Comunidad Económica Europea, contrato ERBIC18CT980317

2 Laboratorio de Fitopatología, Escuela de Ciencias Agrarias, Universidad Nacional. Apdo 86-3000 Heredia. E-mail: caraya@una.ac.cr.

3 Estación Experimental Fabio Baudrit M., Universidad de Costa Rica. Apdo. 183-4050, Alajuela, Costa Rica. E-mail: avillalo@cariari.ucr.ac.cr.
} 
rentes zonas ecológicas, sistemas de siembra, y concentrar en ambos acervos genéticos diferentes genes de resistencia o tolerancia a factores limitantes de la producción. El frijol, cuyas vainas, granos secos, y hojas, pueden ser consumidos en diferentes formas, es una importante fuente de proteína vegetal que mejora la nutrición en situaciones de alto precio de la proteína animal. Su cultivo se encuentra mundialmente, siendo Brasil, México, Estados Unidos y Argentina, los mayores productores (Pachico, 1994).

Una de las principales limitaciones del cultivo de frijol en América Latina es el ataque de enfermedades. Los patógenos más frecuentes, como por ejemplo, Colletotrichum lindemuthianum, Phaeoisariopsis griseola y Uromyces appendiculatus, son organismos en los que se ha identificado una gran variabilidad patogénica, que refleja procesos de coevolución en el patosistema (Araya, 1996, Pastor-Corrales y Jara, 1995, Pastor-Corrales y Otoya, 1995). En Costa Rica, durante los últimos diez años se ha estudiado la variabilidad patogénica de $C$. lindemuthianum, agente causal de la antracnosis del frijol. Los datos demuestran que las poblaciones de este patógeno son más virulentas y su variabilidad es más amplia que la encontrada en otras regiones (Araya y Cárdenas, 1999). Por el contrario, la variabilidad patogénica en mancha angular $(P$. griseola $)$ ha sido menos estudiada; recientemente se informó de la presencia de razas fisiológicas en América Central, donde $60 \%$ de los aislamientos caracterizados correspondió a nuevas razas y los aislamientos colectados en México y Honduras resultaron ser los más virulentos (Jara, 1998).

En relación con la variabilidad del patosistema, en Mesoamérica se concentra la mayor diversidad, no solo del hospedante, sino también del patógeno. Desde la perspectiva del hospedante, esta situación representa una ventaja ya que aumenta la probabilidad de detectar genes de resistencia a las diversas razas del patógeno, dentro del mismo acervo genético. Por tal razón, los programas de mejoramiento de la región deben contemplar la evaluación de la mayor cantidad posible de líneas y cultivares, de los más diversos orígenes, así como el análisis de la respuesta de esas a las poblaciones de los patógenos presentes en cada sitio de evaluación, y prever la presencia de variantes patogénicas en otras regiones (Beebe y Pastor-Corrales, 1991). Al respecto, ocho genes de resistencia ya han sido identificados (Kelly y Young, 1996), siete de los cuales proceden del acervo genético Mesoamericano y cuentan con sus respectivos marcadores moleculares. Pastor-Corrales (1995b) encontró la expresión de algunos de estos genes en 4.939 accesiones de frijol evaluadas en el campo e invernadero con aislamientos colombianos. Sin embargo, estas fuentes de resistencia no son efectivas en América Central por la alta virulencia de las poblaciones nativas del patógeno. Actualmente, no existe información disponible sobre fuentes de resistencia a las razas presentes en la región. En similares condiciones se encuentran los trabajos de búsqueda de genes de resistencia a $P$. griseola.

En Costa Rica se ha trabajado en la selección de genotipos con tolerancia a factores abióticos limitantes y resistencia a enfermedades, principalmente antracnosis, mancha angular y mustia hilachosa (Thantephorus cucumeris). El objetivo del Programa de Investigación y Transferencia de Tecnología Agropecuaria en Frijol [PITTA-Frijol] de Costa Rica es identificar líneas con amplia adaptación a suelos de baja fertilidad y resistencia a los patógenos de mayor relevancia en la región y generar fuentes de resistencia para los programas de mejoramiento de América Central. El presente trabajo expone los resultados de las investigaciones llevadas a cabo en la búsqueda de esas fuentes de resistencia.

\section{MATERIALES Y MÉTODOS}

Líneas de frijol Andino o Mesoamericano, provenientes de diferentes viveros y programas de mejoramiento, fueron evaluadas y seleccionadas en los ciclos 97-98 y 98-99 en Puriscal (1017 msnm) y en las estaciones experimentales Fabio Baudrit (814 msnm) y Fraijanes (1650 msnm). La escogencia de los sitios de siembra obedeció al interés de evaluar los materiales en diferentes climas con condiciones favorables para al menos dos patógenos. Así, Puriscal reúne condiciones que favorecen el desarrollo de antracnosis y mustia, en la estación Fabio Baudrit se desarrolla mejor la mancha angular, mientras que Fraijanes ofrece condiciones de temperatura favorables para el ataque de antracnosis y suelos de baja fertilidad.

Las fuentes de materiales fueron el vivero de fuentes de resistencia (VIFURE) a antracnosis y a baja fertilidad, el vivero de padres donantes de genes necesarios (VIPADOGEN), la colección CORE, todos del Centro Internacional de Agricultura Tropical (CIAT), y un grupo de líneas criollas de Costa Rica y líneas promisorias provenientes de los programas de mejoramiento de Costa Rica y de CIAT. Los materiales fueron sembrados en surcos de dos a seis metros de largo con una o dos repeticiones dependiendo de la disponibilidad de semilla. La densidad de siembra fue 15 semillas/m, cubiertas con inoculante de Rhizobium $(1 \mathrm{~kg} / 100 \mathrm{lb})$. No se aplicó fertilizante adicional ni fungicidas al follaje. 
La evaluación de enfermedades se realizó a las seis u ocho semanas después de la siembra. La reacción de las plantas a antracnosis, mustia hilachosa y mancha angular, se evaluó siguiendo la escala de severidad elaborada por el CIAT (1987), la que consta de nueve grados de severidad, donde una es planta sana y nueve planta muerta. Para asegurar un nivel óptimo de inóculo en el campo, todos los ensayos fueron inoculados artificialmente tres o cuatro veces durante el ciclo del cultivo, con suspensiones de $1,2 \times 10^{6}$ conidios de $C$. lindemuthianum por ml, de las razas 9, 10, 303 y 457. Para efectos de selección, se tomaron como resistentes solo los materiales con reacción de uno a tres para antracnosis y de uno a cuatro para mancha angular.

\section{RESULTADOS Y DISCUSIÓN}

Las poblaciones de $C$. lindemuthianum y P. griseola en Costa Rica son muy variables. En el primer caso se ha observado una variabilidad cercana al $60 \%$, mientras que para el segundo patógeno la variabilidad es del $55 \%$ (PITTA frijol, 1999). Por tal razón, la adecuada selección de fuentes de resistencia demanda el conocimiento de la variabilidad poblacional patógeno, para garantizar la selección de líneas con resistencia al mayor número de razas o variantes presentes en la región. Los resultados de las evaluaciones a enfermedades demostraron que dentro de la diversidad genética contenida en los diferentes viveros, es posible identificar genotipos con resistencia a factores limitantes de la

Cuadro 1. Reacción a enfermedades (escala 1-9) de líneas seleccionadas en el programa de mejoramiento de frijol de Costa Rica, evaluadas a la sexta y octava semanas después de la siembra en Puriscal, Costa Rica, 1998.

\begin{tabular}{lcccccc}
\hline \multicolumn{1}{c}{ Línea } & \multicolumn{2}{c}{ Antracnosis } & Mancha & Angular & \multicolumn{2}{c}{ Mustia } \\
& sexta & octava & sexta & octava & sexta & octava \\
\hline A 931 & 1 & 1 & 1 & 1 & 4 & 7 \\
A 247 & 1 & 4 & 5 & 8 & 6 & 6 \\
A 483 & 1 & 1 & 1 & 6 & 6 & 6 \\
AB 136 & 1 & 1 & 6 & 8 & 5 & 7 \\
AND 277 & 7 & 9 & 1 & 1 & 1 & 1 \\
AND 279 & 1 & 6 & 1 & 1 & 8 & 9 \\
Amendoin & 1 & 1 & 1 & 1 & 8 & 9 \\
CAL 143 & 1 & 1 & 1 & 1 & 8 & 9 \\
Calima & - & - & - & - & 9 & 9 \\
G 205 & 1 & 4 & 1 & 3 & 5 & 7 \\
G 19428 & 1 & 1 & 4 & 1 & 5 & 4 \\
G 11640 & 2 & 2 & 5 & 8 & 5 & 7 \\
G 12517 & 1 & 2 & 4 & 1 & 5 & 7 \\
G 1368 & 1 & 1 & 6 & 1 & 6 & 7 \\
G 15317 & 1 & 2 & 5 & 5 & 6 & 7 \\
G 19404 & 1 & 2 & 5 & 5 & 5 & 7 \\
G 2337 & 1 & 1 & 6 & 4 & 8 & 8 \\
G 4672 & 3 & 2 & 1 & 1 & 5 & 7 \\
G 5686 & 1 & 2 & 1 & 1 & 5 & 7 \\
ANT 12 & 1 & 1 & 1 & 1 & 6 & 7 \\
TF 9223 & 1 & 1 & 1 & 4 & 4 & 4 \\
TIF 1 & 1 & 1 & 4 & 7 & 4 & 6 \\
TLP 17 & 4 & 4 & 4 & 5 & 6 & 7 \\
\hline
\end{tabular}

Cuadro 2. Reacción a enfermedades (escala 1-9) de líneas seleccionadas en el Programa de Mejoramiento de Costa Rica, evaluadas en Alajuela a las siete semanas después de la siembra. Costa Rica, 1998.

\begin{tabular}{lcccc}
\hline \multicolumn{1}{c}{ Línea } & Antracnosis & $\begin{array}{c}\text { Mancha } \\
\text { Angular }\end{array}$ & Mustia & $\begin{array}{c}\text { Mosaico } \\
\text { Dorado }\end{array}$ \\
\hline Manzano & 1 & 5 & 1 & 1 \\
UCR 55 & 1 & 1 & 5 & 1 \\
A 483 & 1 & 1 & 1 & 1 \\
BAC 50 & 4 & 1 & 5 & 1 \\
G 92 & 1 & 5 & 1 & 1 \\
VAX 2 & 6 & 5 & 6 & 7 \\
VAX 2 & 4 & 6 & 1 & 7 \\
G 18515 & 2 & 4 & 6 & 1 \\
V 8025 & 1 & 5 & 6 & 6 \\
A 247 & 1 & 5 & 7 & 1 \\
MAR 3 & 1 & 1 & 1 & 1 \\
N-INIFAP & 4 & 1 & 3 & 7 \\
G 13755 & 1 & 4 & 1 & 1 \\
RAZ 59 & 1 & 7 & 4 & 6 \\
G 11640 & 1 & 1 & 4 & 7 \\
K 2 & 1 & 1 & 8 & 1 \\
Bayo Alteño & 1 & 4 & 1 & 1 \\
G 10843 & 1 & 1 & 6 & 7 \\
AND 277 & 1 & 1 & 1 & 1 \\
A 36 & 1 & 1 & 6 & 7 \\
A 193 & 1 & 1 & 1 & 1 \\
Calima & 1 & 1 & 5 & 1 \\
G 13860 & 1 & 1 & 4 & 7 \\
MOC 112 & 5 & 7 & 7 & 7 \\
G 12529 & 1 & 4 & 1 & 4 \\
Guanajuato 32 & 1 & 4 & 1 & 4 \\
G 19428 & 1 & 1 & 4 & 3 \\
G 19696 & 1 & 1 & 1 & 1 \\
G 19860 & 1 & 1 & 1 & 1 \\
A 321 & 1 & 1 & 6 & 7 \\
\hline
\end{tabular}

producción, tanto bióticos como abióticos. Esa diversidad genética del hospedante es una fuente de genes de resistencia a patotipos, también variables.

Del grupo de 23 genotipos seleccionados en los últimos dos ciclos agrícolas en Puriscal (Cuadro 1), se identificaron materiales con resistencia combinada a antracnosis y a mancha angular. Por ejemplo, las líneas A 931, Amendoin, CAL 143, G 12517, G4672, G5686, G 19428, ANT 12 y TF 9223, mostraron reacción resistente a las poblaciones de ambos patógenos. Por el contrario, cinco líneas presentaron resistencia específica a antracnosis y dos líneas resistencia específica a mancha angular. Cuando parte de estos materiales se sembraron bajo las condiciones de la estación Fabio Baudrit y se evaluaron durante los dos ciclos agrícolas, los resultados mostraron un mayor número de líneas con resistencia a las poblaciones de antracnosis (Cuadro 2). Sin embargo, como se mencionó anteriormente, este sitio es más favorable al desarrollo de mancha angular, por lo que la presión de inóculo es mayor y consecuentemente la reacción de los materiales a este patógeno es más precisa.

Las líneas citadas como resistentes a antracnosis en Puriscal (Cuadro 1) constituyen un grupo amplio de 
fuentes de resistencia de alta estabilidad, que han expresado su reacción a través de más de tres ciclos de evaluación y ante diferentes patotipos. Estas fuentes formarán un vivero específico centroamericano para evaluar en otras condiciones. Además, de acuerdo con las necesidades de hibridación, las fuentes están a disposición de los programas de mejoramiento de la región centroamericana.

El VIPADOGEN, vivero de 74 líneas formado por el CIAT, también fue evaluado en Puriscal y en la estación Fabio Baudrit. Este vivero estuvo constituido por líneas seleccionadas no sólo por su reacción a antracnosis y a mancha angular, sino que también combinan adaptación a condiciones de baja fertilidad o a sequía. En este vivero sólo se detectaron dos líneas (A 222 y A 193) con resistencia a antracnosis y mancha angular. Con resistencia específica a $C$. lindemuthianum se identificaron 23 líneas, algunas de ellas combinando tolerancia a baja fertilidad o a sequía (Cuadro 3). En los datos de cada sitio se observó un mayor número de materiales seleccionables por resistencia a un patógeno,

Cuadro 3. Líneas seleccionadas del VIPADOGEN* por su resistencia a la antracnosis o la mancha angular más un facto abiótico, evaluadas en Puriscal y Alajuela, Costa Rica, 1998

\begin{tabular}{|c|c|}
\hline Antracnosis & Factor Abiótico ** \\
\hline \multicolumn{2}{|l|}{ A 222} \\
\hline \multicolumn{2}{|l|}{ AFR 188} \\
\hline \multicolumn{2}{|l|}{ CNF 5558} \\
\hline \multicolumn{2}{|l|}{ MAR 1} \\
\hline \multicolumn{2}{|l|}{ MAR 2} \\
\hline \multicolumn{2}{|l|}{ MAR 3} \\
\hline \multicolumn{2}{|l|}{ MEX 54} \\
\hline \multicolumn{2}{|l|}{ A 193} \\
\hline \multicolumn{2}{|l|}{ ARA 18} \\
\hline A 195 & Baja fertilidad \\
\hline A 774 & Baja fertilidad \\
\hline Carioca & Baja fertilidad \\
\hline FEB 190 & Baja fertilidad \\
\hline G 2402 & Baja fertilidad \\
\hline G 14665 & Baja fertilidad \\
\hline G 19839 & Baja fertilidad \\
\hline G 22263 & Baja fertilidad \\
\hline \multicolumn{2}{|l|}{ DOR 500} \\
\hline \multicolumn{2}{|l|}{ FEB 209} \\
\hline \multicolumn{2}{|l|}{ G 92} \\
\hline \multicolumn{2}{|l|}{ PEF 13} \\
\hline SEA 7 & Sequía \\
\hline SEA 13 & Sequía \\
\hline Mancha Angular & Factor Abiótico \\
\hline \multicolumn{2}{|l|}{ A 222} \\
\hline \multicolumn{2}{|l|}{ A 193} \\
\hline \multicolumn{2}{|l|}{ G 13772} \\
\hline \multicolumn{2}{|l|}{ ICA Pijao } \\
\hline \multicolumn{2}{|l|}{ Porrillo Sintético } \\
\hline SEA 2 & Sequía \\
\hline
\end{tabular}

* VIPADOGEN= Vivero de padres donantes de genes necesarios

** Factor abiótico identificado por CIAT pero al combinar los datos de ambos sitios, ese número se redujo por reacción diferencial en uno o en otro sitio. Esta respuesta obedece a diferencias en las condiciones ambientales y en la estructura poblacional de los patógenos, situación que es muy frecuente en los programas de selección de líneas promisorias, como lo menciona Pastor-Corrales (1995a, 1995b).

La descriminación de materiales fue más severa en la colección CORE. Del total de 141 genotipos, únicamente 26 mostraron resistencia combinada a antracnosis y a mancha angular (Cuadro 4), aunque el número aumentó significativamente cuando se consideró solo una enfermedad. En este caso, 82 materiales fueron resistentes a antracnosis y 12 lo fueron a mancha angular.

Cuadro 4. Reacción a enfermedades (escala 1-9) de líneas seleccionadas de la colección CORE evaluadas en Puriscal. Costa Rica, 1998

\begin{tabular}{|c|c|c|c|}
\hline Línea & Antracnosis & Mancha Angular & Mustia \\
\hline G 62 & 1 & 1 & 5 \\
\hline A 163 & 3 & 3 & 4 \\
\hline ICA Tundama & 1 & 2 & 6 \\
\hline G 1137 & 1 & 1 & 8 \\
\hline G 1294 & 4 & 1 & 4 \\
\hline Namunye Red & 1 & 1 & 7 \\
\hline IZ 98 & 1 & 1 & 7 \\
\hline PVA 82 & 1 & 1 & 7 \\
\hline Kilyumkwe & 1 & 3 & 7 \\
\hline G 392 & 1 & 1 & 6 \\
\hline G 972 & 1 & 1 & 8 \\
\hline G 610 & 1 & 1 & 7 \\
\hline G 611 & 1 & 1 & 5 \\
\hline G 615 & 1 & 4 & 7 \\
\hline G 621 & 1 & 4 & 7 \\
\hline G 693 & 1 & 1 & 8 \\
\hline G 712 & 1 & 4 & 7 \\
\hline G 732 & 1 & 1 & 8 \\
\hline CF 26 & 1 & 1 & 7 \\
\hline G 775 & 1 & 1 & 8 \\
\hline G 777 & 1 & 1 & 8 \\
\hline G 791 & 1 & 1 & 8 \\
\hline G 837 & 1 & 1 & 7 \\
\hline G 846 & 1 & 1 & 4 \\
\hline G 851 & 1 & 1 & 4 \\
\hline G 992 & 1 & 1 & 8 \\
\hline
\end{tabular}

CORE $=$ Vivero del CIAT.

Con base en los datos obtenidos, es posible identificar genes de resistencia a los patotipos presentes en Costa Rica, dentro de la amplia variabilidad genética que presenta el hospedante, no sólo al interior del acervo Mesoamericano, sino también importando genes del acervo Andino. Pastor-Corrales et al. (1995a, 1995b) consideran que el mayor número de genes de resistencia se encuentran en genotipos de origen opuesto al aislamiento; es decir, es más probable encontrar genes de resistencia a razas mesoamericanas del patógeno en materiales Andinos que en Mesoamericanos. Sin embargo, esta estrategia no es del todo válida para las po- 
blaciones del patógeno en América Central por su mayor virulencia. Dada la amplia variabilidad del acervo mesoamericano (Singh et al., 1991) es posible identificar genes de resistencia a poblaciones autóctonas del patógeno.

Las fuentes de resistencia provenientes de los viveros internacionales, a diferencia de las originadas en los programas de hibridación nacionales (Cuadro 1), requieren de un nuevo ciclo de evaluación para determinar la estabilidad de las mismas en el tiempo. Tienen la virtud de cargar otros genes piramidados que le otorgan tolerancia a diversos factores abióticos, pero su reacción a los patotipos de América Central debe ser comprobado para ser utilizadas en los programas de mejoramiento.

En ninguno de los viveros evaluados se logró determinar materiales con tolerancia a mustia hilachosa, lo que sugiere la necesidad de buscar genes de resistencia en acervos secundarios o terciarios para incorporarlos en los cultivares comerciales.

\section{LITERATURA CITADA}

ARAYA, C.M. 1996. Pathogenic and molecular variability and telia production of Uromyces appendiculatus isolates from the Andean and Middle American centers of domestication of common bean. Ph.D. thesis. University of Nebraska-Lincoln. 159p.

ARAYA, C.M.; CÁRDENAS, C. 1999. Variabilidad patogénica y fuentes de resistencia a poblaciones de Colletotrichum lindemuthianum en Costa Rica. Resúmenes XXXIX Reunión Anual APS-CD. San Juan, Puerto Rico. p.72.

BEEBE, S.E.; PASTOR-CORRALES, M.A. 1991. Breeding for disease resistance. In: A. van Schoonhoven and O. Voysest. Common beans; Research for crop improvement. CIAT, Cali, Colombia. 980p.
CIAT. 1987. Sistema estándar para la evaluación de germoplasma de frijol. Art van Schoohoven y Marcial PastorCorrales (comp). Cali, Colombia. 56 p.

GEPTS, P; DEBOUCK, D. 1991. Origen, Domestication and Evolution of the common bean (Phaseolus vulgaris L.). In: A. Van Schoohoven and O. Voy sest (Eds.). CIAT. Cali, Colombia. p 7-54.

JARA, C. 1998. Diversidad patogénica de Phaeoisariopsis griseola centroamericana, causante de la mancha angular del frijol comúm. Resúmenes XLIV Reunión Anual PCCMCA. .Man., Nicaragua. p.102.

PACHICO, D. 1994. Tendencias en la producción mundial de frijol común. In: M.A. Pastor-Corrales y H.F. Schwartz (Eds.) Problemas de producción del frijol en los trópicos. CIAT. Cali, Colombia. pp1-10.

PASTOR-CORRALES, M.A; JARA, C. 1995a. La evolución de Phaeoisariopsis griseola con el frijol común en América Latina. Fitop. Col. 19:15-24.

PASTOR-CORRALES, M.A.; OTOYA, M.M.; MOLINA, A.; SINGH, S.P. 1995b. Resistance to Colletotrichum lindemuthianum isolates from Middle America and Andean South America in different common bean races. Plant Dis. 79:63-67.

PITTA FRIJOL (Programa de Investigación y Transferencia de Tecnología Agropecuaria en FrijoL). 1999. Informe Técnico Anual 1998-1999. S.J., Costa Rica. pp 42-43.

SINGH, S.P.; GUTIÉRREZ, J.A; MOLINA, A.; URREA, C.; GEPTS, P. 1991. Genetic diversity in cultivated common bean: II Marker-based analysis of morphological and agronomic traits. Crop Sc. 31:23-29.

YOUNG, R.A; KELLY, J.D. 1996. Characterization of the genetic resistance to Colletotrichum lindemuthianum common bean differential cultivars. Plant Disease 80: 650-654. 